\title{
Dyslipidemia and Intraperitoneal Inflammation Axis in Peritoneal Dialysis Patients: A Cross-Sectional Pilot Study
}

\author{
Natalia Stepanova ${ }^{\text {a }}$ Victoria Driianska ${ }^{b}$ Svitlana Savchenko ${ }^{a}$

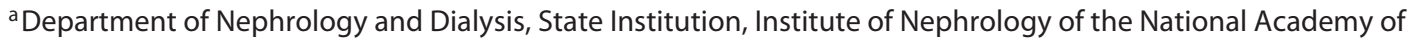 \\ Medical Sciences, Kyiv, Ukraine; ${ }^{b}$ Immunology Laboratory, State Institution, Institute of Nephrology of the National \\ Academy of Medical Sciences, Kyiv, Ukraine
}

\section{Keywords}

Dyslipidemia · Intraperitoneal inflammation · Peritoneal dialysis

\begin{abstract}
Background: We have hypothesized that the problem of dyslipidemia in peritoneal dialysis (PD) patients lies beyond certain levels of plasma lipoprotein and involves cardiovascular risk, but can also influence the development of chronic intraperitoneal inflammation. Objectives: The aim of our work was to define whether the association of dyslipidemia with intraperitoneal inflammation really exists and if it could it be used in a prospective cohort of PD patients. Patients and Methods: We performed a cross-sectional, single-center, pilot study involving 40 nondiabetic PD patients ( 27 men and 13 women with an average age of $49.3 \pm 12.2$ years). The median time on PD was 29 (18.5-37) months. The parameters dialysis adequacy, blood lipid profile, and the concentrations of tumor necrosis factor (TNF)-a, monocyte chemoattractant protein (MCP)-1, and interleukin (IL)-10 in peritoneal dialysate effluent (PDE) were determined. Cohen's $d$ effect size was computed post hoc to determine the differences between groups in the concentrations of pro- and anti-inflammatory mediators. Results: PD patients with atherogenic dyslipidemia had significantly high levels of MCP-1 com-
\end{abstract}

pared with dyslipidemia-free patients (Cohen's $d=1.32$ ). A reduced high-density lipoprotein cholesterol level was associated with a high intraperitoneal production of the proinflammatory mediator TNF-a $(p<0.0001)$ and anti-inflammatory IL-10 ( $p<0.0001)$. Atherogenic index of plasma was directly correlated with MCP-1 $(p<0.0001)$ and TNF-a $(p<$ $0.0001)$. In multiple regression analysis, MCP-1 appeared to predict PD inadequacy $\left(R^{2}=0.58 ; F\right.$ ratio $\left.=9.4 ; p=0.006\right)$ independently of age and blood $C$-reactive protein level. Effect size was 1.38 with $a=0.05, n=40$, and 3 predictors. Conclusions: Our cross-sectional pilot study first demonstrated a close interaction between the atherogenic lipid profile and a high concentration of MCP-1 in PDE; this might be a prognostic marker for PD inadequacy. The potential significance of our finding is that it provides useful preliminary information necessary for further research into this area.

\footnotetext{
(C) 2019 The Author(s)

Published by S. Karger AG, Base
}

\section{Introduction}

It is a well-known fact that patients with peritoneal dialysis (PD) have lipid profiles that are more atherogenic than in nondialyzed and hemodialysis patients [1-3]. Both the protein loss in the dialysate and the use of glu-

\begin{tabular}{ll}
\hline KARGER & $\begin{array}{l}\text { ( } 2019 \text { The Author(s) } \\
\text { Published by S. Karger AG, Basel }\end{array}$ \\
E-Mail karger@karger.com & $\begin{array}{l}\text { This article is licensed under the Creative Commons Attribution- } \\
\text { NonCommercial-NoDerivatives 4.0 International License (CC BY- } \\
\text { NC-ND) (http://www.karger.com/Services/OpenAccessLicense). } \\
\text { Usage and distribution for commercial purposes as well as any dis- } \\
\text { tribution of modified material requires written permission. }\end{array}$
\end{tabular}

Natalia Stepanova, MD, PhD, Dr.Sc

Head of Department of Nephrology and Dialysis

State Institution, Institute of Nephrology of the National Academy of Medical Sciences Degtyarivska 17 V, Kyiv 04050 (Ukraine)

E-Mail nmstep88@gmail.com 
cose-based solutions might contribute to this condition $[4,5]$. Moreover, PD treatment leads to body weight gain and can be a potential cause of accelerated atherosclerosis and chronic inflammation [6-8]. In turn, chronic inflammation is closely associated with impaired nutritional status and peritoneal membrane and PD technique failure [7].

We hypothesize that the problem of dyslipidemia in $\mathrm{PD}$ patients is beyond certain levels of plasma lipoprotein and involves cardiovascular (CV) risk but can also influence the development of chronic intraperitoneal inflammation. Recent clinical and experimental studies have demonstrated the crucial role of high production of tumor necrosis factor (TNF)- $\alpha$ and monocyte chemoattractant protein (MCP)-1 in peritoneal fibrosis [9-11]. In response to TNF- $\alpha$ secreted by peritoneal macrophages, peritoneal mesothelial cells synthesize various cytokines and thus enhance the inflammatory signal $[9,12]$. The main function of MCP-1 is to attract peritoneum monocytes/macrophages and their activation [13]. It has also been demonstrated that TNF- $\alpha$ and MCP-1 are atherosclerosis-promoting chemokines, and that cytokines with anti-inflammatory activity (including interleukin [IL]10) released into the abdominal cavity suppress the expression of numerous proinflammatory mediators and reduce the influx of macrophages [14-16]. However, there are no reports available on the effects of dyslipidemia on intraperitoneal inflammation and PD adequacy.

We performed a pilot study on PD patients, with the following aims: (1) to characterize TNF- $\alpha$, MCP-1, and IL-10 dialysate levels; (2) to define whether the association of dyslipidemia with intraperitoneal inflammation really exists; (3) to determine the ways this could be used in a prospective cohort study in PD patients. The study hypothesis was that atherogenic lipid profile correlated with pro- and anti-inflammatory mediators in peritoneal dialysate effluent (PDE) and, respectively, PD adequacy.

\section{Patients and Methods}

\section{Study Design and Subjects}

This cross-sectional, single-center, pilot study is part of an ongoing project which is being run by the State Institution "Institute of Nephrology of the National Academy of Medical Sciences" in Kyiv, Ukraine. The study involved 40 nondiabetic PD patients who had been treated with continuous ambulatory peritoneal dialysis (CAPD) for $\geq 3$ months with the usual hospital stay (4-5 h during the day and $8-10 \mathrm{~h}$ at night). The duration of PD therapy at this study entry (at baseline) was 29 (18.5-37) months. All patients received commercially available, glucose-based Dianeal PD solution (Baxter Inc.)) of various strengths (1.36 and 2.27\%), and Icodex- trine. We excluded the patients with malignant diseases, diabetes, a history of peritonitis, or a significant illness/hospitalization in the previous 3 months.

\section{Clinical and Laboratory Measurements}

Patient demographics including age, gender, comorbid conditions, hypertension, and medicine use were obtained from the medical records of the patients at our hospital. Laboratory tests were evaluated during the clinic visit at the time of PDE collection for measurement of immune mediators.

Whole-blood samples were collected from the patients after an overnight fast during the routine outpatient visit. The blood samples were processed immediately after sampling.

Routine biochemical parameters including blood and daily dialysate concentration of urea and creatinine, serum albumin, Creactive protein (CRP), glucose, electrolytes, and lipid profile parameters were carried out using an automatic analyzer (Flexor Junior, Vital Scientific, Spankeren, The Netherlands). Hematological parameters of blood were determined using an ABX Micros-60 analyzer (Viollier, France).

Blood lipid profile parameters included triglyceride (TG), total cholesterol (TC), high-and low-density lipoprotein cholesterol (HDL-C and LDL-C), and very low-density lipoprotein cholesterol (VLDL-C). The atherogenic index of plasma (AIP) was calculated from plasma triglyceride and HDL-C (log [TG/HDL-C]). Atherogenic dyslipidemia was defined as a combination of low plasma levels of LDL-C $\geq 2.59 \mathrm{mmol} / \mathrm{L}$ and elevated $\mathrm{TG} \geq 2.26$ $\mathrm{mmol} / \mathrm{L}$, according to the K/DOQI clinical practice guidelines for managing dyslipidemia in chronic kidney disease [17]. Body mass index (BMI) was also calculated.

Dialysis adequacy was determined by measuring total weekly creatinine clearance $(\mathrm{CrCl})$ (normalized to $1.73 \mathrm{~m}^{2}$ of the body surface area) and total weekly urea clearance (Kt/V) using the Watson formula for body water [18]. Peritoneal Kt/V, plasma Kt/V, and renal Kt/V were estimated separately. The dialysate-to-plasma creatinine ratio $(\mathrm{D} / \mathrm{P})$ was calculated from the concentrations of creatinine in the 24-h dialysate and plasma; we used a standardized procedure for peritoneal equilibration test (PET) proposed by Twardowski et al. [19].

\section{Immune Mediators Measurements in PDE}

All samples for biomarker assessment were obtained from the overnight PDE (from exchanges with 2 L 1.36 or $2.27 \%$ glucose dialysate) before the test, and then stored at $-80^{\circ} \mathrm{C}$ until analysis.

The TNF- $\alpha$, MCP-1, and IL-10 concentrations were detected in PDE using STAT FAX-303 PLUS and commercially available enzyme-linked immunosorbent assay (ELISA) test kits (Diaclon, France; DRG, Germany; Ukrmedservice, Ukraine) according to the manufacturers' protocols.

\section{Statistical Analysis}

Analysis and all graphs were performed using MedCalc (Belgium). The average mean and $\mathrm{SD}$ or the median and interquartile range (Q25-Q75) were calculated according to a normal distribution. For the statistical analysis, we used the Student $t$ test and nonparametric Mann-Whitney U test.

Cohen's $d$ effect size was computed post hoc to determine the differences in pro- and anti-inflammatory mediator concentrations between the groups (no a priori power analysis was possible because pilot data were not available). Post hoc power analysis was 
Table 1. Demographic and clinical characteristics of the study participants according to atherogenic dyslipidemia status

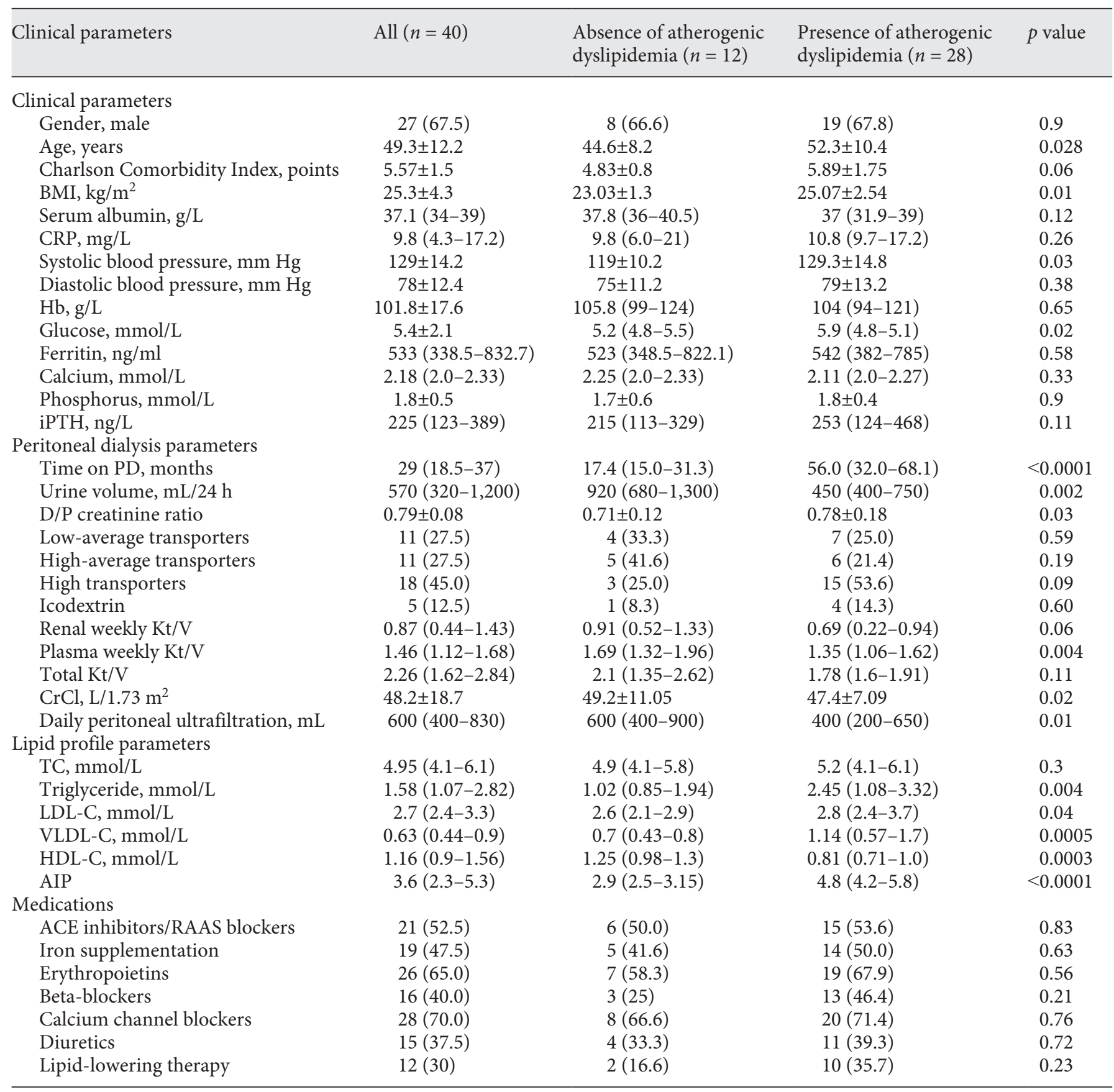

The values are expressed as mean \pm standard deviation $(\mathrm{M} \pm \mathrm{SD})$ or as median and interquartile range (Median $(\mathrm{Q} 25-\mathrm{Q} 75)$ ) or $n(\%)$. The values are compared between the groups using the chi-square test, $t$ test and Mann-Whitney U test as appropriate. AIP, atherogenic index of plasma; ACE, angiotensin-converting enzyme; $\mathrm{BMI}$, body mass index; $\mathrm{CrCl}$, creatinine clearance; CRP, C-Reactive Protein; Hb, hemoglobin; HDL-C, high-density lipoprotein cholesterol; iPTH, intact parathyroid hormone; LDL-C, low-density lipoprotein cholesterol; RAAS, renin-angiotensin-aldosterone system; TC, total cholesterol; total Kt/V, total weekly Kt/Vurea; VLDL-C, very low density lipoprotein cholesterol. 
Table 2. PDE pro- and anti-inflammatory markers related to dyslipidemia patients's status

\begin{tabular}{lcllll}
\hline Immune mediators & All $(n=40)$ & $\begin{array}{l}\text { Patients without } \\
\text { dyslipidemia }(n=12)\end{array}$ & $\begin{array}{l}\text { Patients with } \\
\text { dyslipidemia }(n=28)\end{array}$ & $\begin{array}{c}p \text { value } \\
\text { Cohen's } d \\
\text { effect size }\end{array}$ & $\begin{array}{l}\text { Power } \\
(1-\beta)\end{array}$ \\
\hline IL-10, pg/mL, median (IQR) & $7.5(5.9-145.0)$ & $3.0(2.9-3.0)$ & $21.8(5.2-86.2)$ & 0.0002 & 0.03 \\
MCP-1, pg/mL, mean \pm SD & $20.7 \pm 9.2$ & $17.7 \pm 9.9$ & $29.3 \pm 8.6$ & 0.002 & 1.32 \\
TNF- $\alpha, p g / m L$, mean \pm SD & $6.9 \pm 4.0$ & $4.89 \pm 2.8$ & $7.6 \pm 3.7$ & 0.0004 & 0.45 \\
\hline
\end{tabular}

The values are expressed as mean \pm SD or as median and interquartile range (Me (Q25-Q75)). The values are compared between the groups using the chi-square test, $t$ test and Mann-Whitney U test as appropriate. IL-10, interleukin 10; MCP-1, monocyte chemoattractant protein -1; TNF- $\alpha$, tumor necrosis factor-alpha.

performed using $\mathrm{G}^{*}$ Power software v3.1.9.4. The effect size calculators for 2 independent groups (mean \pm SD) with different sample sizes and nonparametric Mann-Whitney U test were used according to a normal distribution [20]. In addition, due to the small statistical power in PDE TNF- $\alpha$ concentrations in the different groups, we also calculated required sample sizes using post hoc Cohen's $d$ values for future large-scale research.

Categorical variables were expressed as proportions and the $\chi^{2}$ test was used for the comparison of 2 groups. Pearson's or Spearman's (as appropriate) correlation test was used to evaluate relationships of immune mediators with lipid profile biomarkers and $\mathrm{PD}$ adequacy parameters.

A multiple linear regression (step-wise method) was fitted to assess possible predictors of low PD adequacy. The AIP and IL-10 concentration values were transformed before inclusion into the model because they were nonnormally distributed. Cohen's $f^{2}$ was used to calculate effect size in the regression analysis according to the following conventions: small effect for values $<0.15$, moderate effect for values 0.15-0.34, and large effect for values $>0.35$ [20].

The null hypothesis was rejected if the $p$ value was $<0.05$.

\section{Results}

\section{Basic Data on Patients}

Forty patients ( 27 men and 13 women) were enrolled in this study at our center. Their mean age was $49.3 \pm 12.2$ years and median time on PD was 29 (18.5-37) months. Demographic and clinical characteristics of the study participants are presented in Table 1. All data were obtained during routine clinical practice immediately after study enrollment.

Dyslipidemia was identified in $70 \%$ of the patients (28/40), although most of them $(18 / 28,64.3 \%)$ did not take lipid-modifying agents (Table 1 ). The subjects without atherogenic dyslipidemia were younger and less obese. They also had lower systolic blood pressure and better glucose control than the patients with atherogenic dyslipidemia. Moreover, BMI ratios were directly correlated with VLDL-C levels $(r=0.42, p=0.007)$.
In addition, there were significant differences between groups in the duration of $\mathrm{PD}$ treatment, daily urine output, $\mathrm{D} / \mathrm{P}$ creatinine ratio, plasma weekly $\mathrm{Kt} / \mathrm{V}$, weekly creatinine clearance, and daily peritoneal ultrafiltration. It is important to note that the PD patients with atherogenic dyslipidemia had significantly lower levels of diuresis $(p=0.002)$, daily peritoneal ultrafiltration $(p=0.01)$, plasma weekly Kt/V $(p=0.004)$, and weekly creatinine clearance $(p=0.02)$ compared with the dyslipidemia-free patients (Table 1).

\section{Analysis of Cytokine Profiles in PDE}

The average levels of IL-10, MCP-1, and TNF- $\alpha$ in PDE were 7.5 (5.9-145.0), 19.7 (14.0-24.8), and 7.6 (5.0513.4) $\mathrm{pg} / \mathrm{mL}$, respectively. PD patients with atherogenic dyslipidemia had significantly high levels of IL-10 and MCP-1 compared with dyslipidemia-free patients (Table 2).

However, it should be noted that while a $d$ value of 1.32 was found for the comparison of MCP-1 between the groups, a small effect size (0.45) was observed for the comparison of TNF- $\alpha$. Besides, no significant effect size was defined for detecting the difference between IL-10 concentrations, indicating a false-positive result. A minimum sample size of 82 participants was required to demonstrate a statistically significant difference in TNF- $\alpha$, with an a value of 0.05 (two-tailed), a medium effect size, and a power (1- $\beta$ error probability) of 0.80 .

\section{Correlational Analysis of Cytokine Profiles in PDE}

Spearman's correlation analysis was performed to investigate the association of the lipid profile with the examined pro- and anti-inflammatory mediators. A reduced HDL-C level was associated with a high intraperitoneal production of the proinflammatory mediators TNF- $\alpha(r=-0.69 ; p<0.0001$; Fig. 1a) and anti-inflammatory IL-10 ( $r=-0.76$; $p<0.0001$; Fig. $1 \mathrm{~b})$. AIP was 


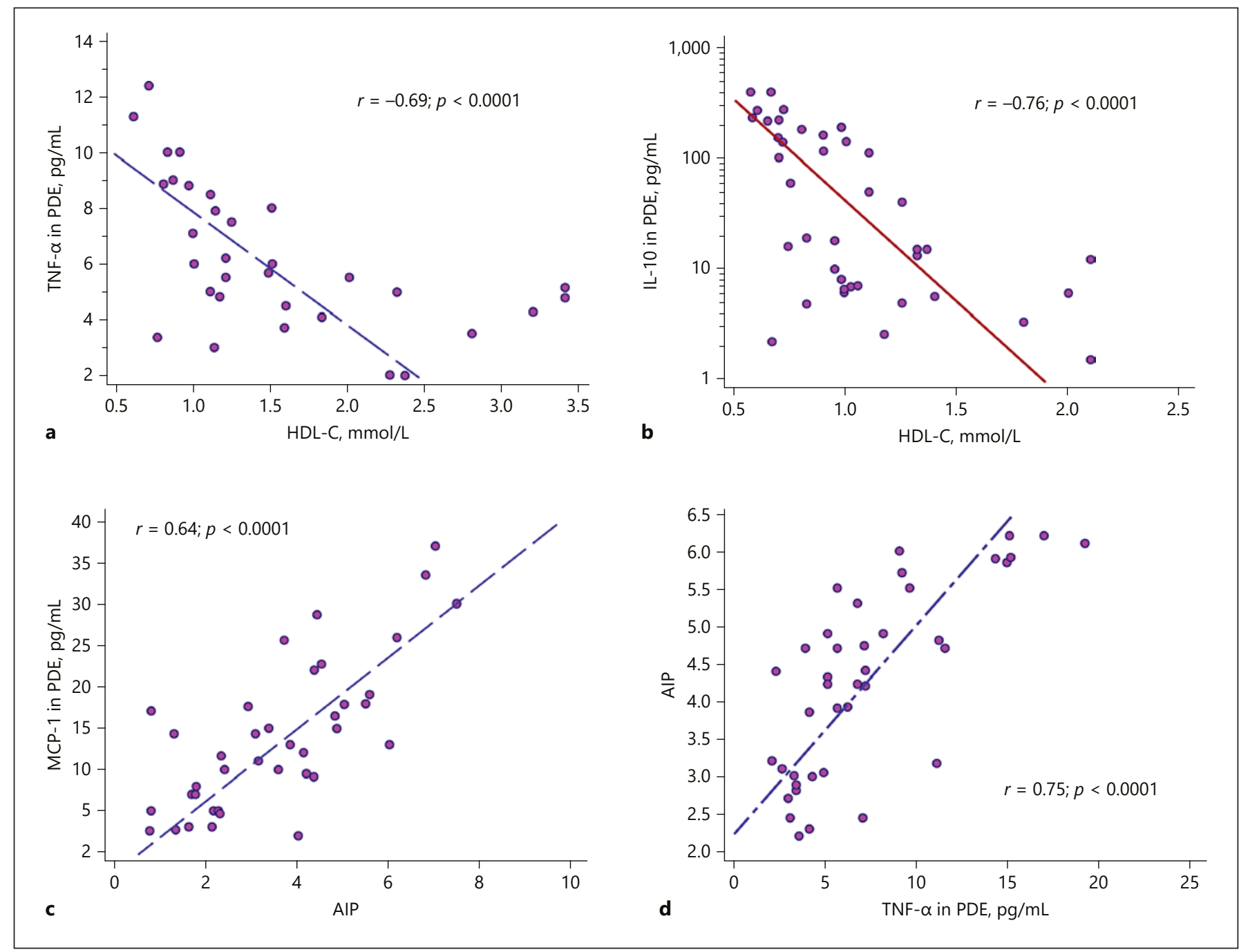

Fig. 1. The correlation between blood lipid profile markers and dialysate immune mediators in PD patients. a The correlation between HDL-C level and TNF- $\alpha$ in PDE in PD patients. $\mathbf{b}$ The correlation between HDL-C level and IL-10 in PDE in PD patients. c The correlation between AIP levels and MCP-1 in PDE in PD patients. d The correlation between AIP levels and TNF- $\alpha$ in PDE in PD patients.

directly correlated with MCP-1 $(r=0.64 ; p<0.0001$; Fig. 1c) and TNF- $\alpha(r=0.75 ; p<0.0001$; Fig. 1d).

There was no statistical association of the examined immune mediators with each other (Table 3 ) or with other lipid profile parameters (data not shown).

Univariate and multiple linear regression analysis were performed for the examined cytokines, to evaluate their influence on dialysis adequacy. In the univariate analysis, the high intraperitoneal production of both TNF- $\alpha$ and MCP- 1 was found to be the explanatory factor for low dialysis adequacy in PD patients with dyslipidemia (Table 4).
In multiple regression, only MCP-1 appeared to predict PD inadequacy (partial correlation coefficient $R^{2}=$ $0.58 ; F$ ratio $=9.4 ; p=0.006$ ), independently of age and blood CRP level; TNF- $\alpha$ did not demonstrate significance in this analysis.

Post hoc power analysis was conducted to determine the risk of a type II error. Effect size for multiple linear regression analysis with $\alpha=0.05, n=40, R^{2}=0.58$, and 3 predictors was 1.38 . Power ( $1-\beta$ error probability) was predicted at 0.99 , suggesting an adequate sample size to observe meaningful relationships among the factors. 
Table 3. The correlations of examined immune mediators between each other

\begin{tabular}{llll}
\hline & IL-10 & MCP-1 & TNF- $\alpha$ \\
\hline IL-10 & $* * *$ & $r=-0.31$ & $r=0.36$ \\
& & $p=0.23$ & $p=0.15$ \\
\hline MCP-1 & $r=-0.31$ & $* * *$ & $r=0.29$ \\
& $p=0.23$ & & $p=0.44$ \\
\hline TNF- $\alpha$ & $r=0.36$ & $r=0.29$ & $* * *$ \\
& $p=0.15$ & $p=0.44$ & \\
\hline
\end{tabular}

IL-10, interleukin 10; MCP-1, monocyte chemoattractant protein -1; TNF- $\alpha$, tumor necrosis factor-alpha.

Table 4. Multiple linear regression analysis of PDE cytokines associated with low PD adequacy

\begin{tabular}{lllr}
\hline Cytokines in PDE & Standardized beta & SE of beta & $p$ value \\
\hline MCP-1 & 0.58 & 0.13 & $<0.0001$ \\
TNF- $\alpha$ & 0.4 & 0.1 & 0.0001 \\
\hline
\end{tabular}

MCP-1, monocyte chemoattractant protein-1; SE, standard error; TNF- $\alpha$, tumor necrosis factor-alpha.

\section{Discussion}

The problem of dyslipidemia observed in PD patients and its effect on cardiovascular and all-cause mortality risks has been well described in previous studies $[1,5$, 21-24]. However, specific reports on the association of lipid metabolism disorders and immune response in $\mathrm{PD}$ patients are limited $[11,25,26]$. Moreover, the strict link between dyslipidemia and chronic intraperitoneal inflammation has never been demonstrated before. To our knowledge, this study is the first cohort study to describe a significant association between the atherogenic lipid profile and a high intraperitoneal production of pro- and anti-inflammatory mediators in PD patients.

In this pilot study, we investigated the possible association of lipid abnormalities with intraperitoneal inflammation and PD efficiency. There are 2 main findings. First, the PDE MCP-1 level was found to be higher in patients with dyslipidemia in comparison with dyslipidemia-free patients. Second, MCP-1 was found to be an independent determinant of PD inadequacy. Post hoc analysis indicated that the study was adequately powered to detect the differences mentioned above.
The association of dyslipidemia with daily peritoneal ultrafiltration and residual renal function in PD patients has been demonstrated in previous studies [27-29]. Specifically, it has been reported that high-transporter PD patients were at increased risk of atherosclerosis from chronic inflammation [29]. In addition, the relationship between the decline of HDL-C and the deterioration of residual renal function has been described [27, 28]. In accordance with these reports, we showed a significantly lower $\mathrm{D} / \mathrm{P}$ creatinine ratio, daily peritoneal ultrafiltration, and urine output in the dyslipidemia condition. Accordingly, a statistically significant decrease of weekly plasma Kt/V and creatinine clearance were observed in our PD patients with an atherogenic lipid profile when compared to the dyslipidemia-free patients.

Available data have reported the results of a crucial role of IL-10, TNF- $\alpha$, and MCP- 1 in atherogenesis $[11,14$, $30]$. On the other hand, these mediators are involved in accelerated insulin resistance and peritoneal fibrosis $[16$, $31,32]$. This particular problem became the basic reason for our initial selection of this cytokine panel.

It has been demonstrated that IL-10 can inhibit effect in atherosclerotic lesions $[11,33]$. Its inactivation accelerates atherosclerosis due to an increased infiltration of inflammatory cells and the production of proinflammatory cytokines [34]. In parallel with this evidence, in our study, a strong inverse correlation of the intraperitoneal production of IL-10 and blood HDL-C level in PD patients was also found. However, the post hoc power analysis did not confirm a significant difference in PDE IL- 10 concentration in our sample.

TNF- $\alpha$ is known to be a multifunctional cytokine implicated in cell homeostasis and immune response regulation in various infectious states, lipid metabolism, and insulin resistance disorders $[11,35]$. TNF- $\alpha$ has been also found to play a key role in developing and accelerating the progression of atherosclerosis $[35,36]$. Earlier, Espinoza et al. [37] observed a positive linear correlation between TNF- $\alpha$ and plasma triglycerides in PD patients. They also described a loss of residual renal function in the hypertriglyceridemia condition. In our study, we did not find the association between TNF- $\alpha$ concentration in PDE and TG level. Nevertheless, we demonstrated a strong direct correlation of dialysate TNF- $\alpha$ level with AIP and an inverse correlation with HDL-C level. Furthermore, a small effect size (0.45) was observed for the comparison of TNF- $\alpha$ level in PDE between the patients with dyslipidemia and the dyslipidemia-free group. In our opinion, the small statistical power of the modest sample size $(n=40)$ could have played a role in limiting the statistical signifi- 
cance. Post hoc power analysis showed that approximately 82 participants would be needed for obtaining statistical power at the recommended 0.80 level.

MCP-1 plays a proinflammatory and atherogenic role in the pathogenesis of atherosclerosis [15]. MCP-1 has also been suggested to have a functional role in the initiation and progression of PD-related epithelial-mesenchymal transition of peritoneal mesothelial cells $[31,37,38]$. Thus, it is obvious that a high concentration of MCP-1 might affect PD adequacy parameters. In our previous reports, we illustrated the positive association between dialysate MCP1 and $\mathrm{PD}$ technique survival in a general nondiabetic PD patient population $[39,40]$. Taking into consideration that this study had a pilot design, we did not assert the unequivocal impact of dyslipidemia on intraperitoneal inflammation in PD patients. We suggest that there was a strong relationship between atherogenic dyslipidemia condition and high dialysate concentration of MCP-1 in this population. This finding is in agreement with other investigations that showed the direct correlation between MCP-1 concentration in dialysate and $\mathrm{D} / \mathrm{P}$ creatinine ratio [41], intraperitoneal inflammation in noninfected PD patients [42], and an increase in all-cause mortality [13].

\section{Limitations}

The interpretation of our results should be taken into account as our study has several limitations. First, it was a small-sample, pilot study performed in a single center, so our findings only revealed associations. Second, levels of cytokines were measured only once at enrollment. Third, we did not evaluate blood concentrations of cytokines. The analysis of the blood/dialysate cytokine ratio and correlation with the lipid profile will allow further insights in connection with the influence of dyslipidemia on subclinical intraperitoneal inflammation in PD patients. It is also important to stress that it was a crosssectional study, and, accordingly, causality could not be established. Despite these limitations, the associations observed indicate the potential contribution of atherogenic dyslipidemia in the development of local subclinical intraperitoneal inflammation.

\section{Conclusions}

In summary, our cross-sectional, pilot study first demonstrated a close interaction between atherogenic lipid profile and a high concentration of MCP-1 in PDE, which could be a prognostic indicator for PD inadequacy. However, bearing in mind that the results did not include a representative sample of PD patients, well-designed clinical trials will be required to establish the impact of dyslipidemia on PD adequacy.

Nonetheless, we suppose that the potential significance of our study is that it provides useful preliminary information necessary for further research in this area and may serve as the basis for future power calculations for large-scale research.

\section{Statement of Ethics}

The study was carried out in accordance with the Declaration of Helsinki, and was the framework of the institute's research work: "The Detection of New Prognostic Negative Determinants of Peritoneal Dialysis Survival and Possible Ways of Their Pharmacological Correction" (Domestic Trial Registration No. 0117U002122). The study protocol was confirmed by the ethics committee of the institute. Written informed consent was obtained from all the subjects participating in the study.

\section{Disclosure Statement}

The authors have no conflicts of interest to declare.

\section{Funding Sources}

This research did not receive any specific grant from funding agencies in the public, commercial, or not-for-profit sectors.

\section{Author Contributions}

N.S. analyzed and interpreted the patient data and was the major contributor in writing the manuscript. V.D. performed the immunological examination. S.S. performed the laboratory tests.

\section{References}

1 Mikolasevic I, Žutelija M, Mavrinac V, Orlic L. Dyslipidemia in patients with chronic kidney disease: etiology and management. Int J Nephrol Renovasc Dis. 2017 Feb;10:35-45.

2 Omran J, Al-Dadah A, Dellsperger KC. Dyslipidemia in patients with chronic and endstage kidney disease. Cardiorenal Med. 2013 Oct;3(3):165-77.

3 Chiu YW, Mehrotra R. Can we reduce the cardiovascular risk in peritoneal dialysis patients? Indian J Nephrol. 2010 Apr;20(2):5967.

4 Guo Q, Lin J, Li J, Yi C, Mao H, Yang X, et al. The effect of fluid overload on clinical outcome in Southern Chinese patients undergoing continuous ambulatory peritoneal dialysis. Perit Dial Int. 2015 Dec;35(7):691-702. 
5 Tsimihodimos V, Mitrogianni Z, Elisaf M. Dyslipidemia associated with chronic kidney disease. Open Cardiovasc Med J. 2011;5(1): 41-8.

6 Rincón Bello A, Bucalo L, Abad Estébanez S, Vega Martínez A, Barraca Núñez D, Yuste Lozano C, et al. Fat tissue and inflammation in patients undergoing peritoneal dialysis. Clin Kidney J. 2016 Jun;9(3):374-80.

7 de Mattos AM, Ovidio PP, Jordão AA, da Costa JA, Chiarello PG. Association of body fat with inflammation in peritoneal dialysis. Inflammation. 2013 Jun;36(3):689-95.

8 Fernström A, Hylander B, Moritz A, Jacobsson H, Rössner S. Increase of intra-abdominal fat in patients treated with continuous ambulatory peritoneal dialysis. Perit Dial Int. 1998 Mar-Apr;18(2):166-71.

9 Lambie M, Chess J, Donovan KL, Kim YL, Do JY, Lee HB, et al.; Global Fluid Study Investigators. Independent effects of systemic and peritoneal inflammation on peritoneal dialysis survival. J Am Soc Nephrol. 2013 Dec; 24(12):2071-80.

10 Wakabayashi K, Hamada C, Kanda R, Nakano T, Io H, Horikoshi S, et al. Adipose-derived mesenchymal stem cells transplantation facilitate experimental peritoneal fibrosis repair by suppressing epithelial-mesenchymal transition. J Nephrol. 2014 Oct;27(5):507-14.

11 Stenvinkel P, Chung SH, Heimbürger O, Lindholm B. Malnutrition, inflammation, and atherosclerosis in peritoneal dialysis patients. Perit Dial Int. 2001;21 Suppl 3:S15762.

12 Maksic D, Vasilijic S, Colic M, StankovicPopovic V, Bokonjic D. Systemic and intraperitoneal proinflammatory cytokine profiles in patients on continuous ambulatory peritoneal dialysis. Adv Perit Dial. 2009;25:50-5.

13 Ko KI, Park KS, Lee MJ, Doh FM, Kim CH, $\mathrm{Koo} \mathrm{HM}$, et al. Increased dialysate MCP-1 is associated with cardiovascular mortality in peritoneal dialysis patients: a prospective observational study. Am J Nephrol. 2014;40(4): 291-9.

14 Methe H, Weis M. Atherogenesis and inflammation - was Virchow right? Nephrol Dial Transplant. 2007 Jul;22(7):1823-7.

15 Lin J, Kakkar V, Lu X. Impact of MCP-1 in atherosclerosis. Curr Pharm Des. 2014; 20(28):4580-8.

16 Strippoli R, Moreno-Vicente R, Battistelli C, Cicchini C, Noce V, Amicone L, et al. Molecular Mechanisms Underlying Peritoneal EMT and Fibrosis. Stem Cells Int. 2016;2016: 3543678.

17 Kidney disease: improving global outcomes (KDIGO) lipid work group. KDIGO clinical practice guideline for lipid management in chronic kidney disease. Kidney Int. 2013;3: 259-305.
18 Ronco C. Adequacy of peritoneal dialysis is more than Kt/V. Nephrol Dial Transplant. 1997;12(1 Suppl 1):68-73.

19 Twardowski ZJ, Prowant BF, Moore HL, Lou LC, White E, Farris K. Short peritoneal equilibration test: impact of preceding dwell time. Adv Perit Dial. 2003;19:53-8.

20 Faul F, Erdfelder E, Lang AG, Buchner A. $\mathrm{G}^{*}$ Power 3: a flexible statistical power analysis program for the social, behavioral, and biomedical sciences. Behav Res Methods. 2007 May;39(2):175-91.

21 Lee MJ, Park JT, Han SH, Kim YL, Kim YS, Yang CW, et al. The atherogenic index of plasma and the risk of mortality in incident dialysis patients: results from a nationwide prospective cohort in Korea. PLoS One. 2017 May;12(5):e0177499.

22 De Vuono S, Ricci MA, Mannarino MR, Lupattelli G. Dyslipidemias and chronic kidney disease: a focus on pathogenesis and treatment. Clin Lipidol. 2014;9(6):673-81.

23 Moradi H, Streja E, Vaziri ND. ESRD-induced dyslipidemia-Should management of lipid disorders differ in dialysis patients? Semin Dial. 2018 Jul;31(4):398-405.

24 Habib AN, Baird BC, Leypoldt JK, Cheung AK, Goldfarb-Rumyantzev AS. The association of lipid levels with mortality in patients on chronic peritoneal dialysis. Nephrol Dial Transplant. 2006 Oct;21(10):2881-92.

25 Oncel M, Akbulut S, Toka Ozer T, Kiyici A, Keles M, Baltaci B, et al. Cytokines, adipocytokines and inflammatory markers in patients on continuous ambulatory peritoneal dialysis and hemodialysis. Ren Fail. 2016 Aug;38(7): 1071-5.

26 Nusair MB, Rajpurohit N, Alpert MA. Chronic Inflammation and Coronary Atherosclerosis in Patients with End-Stage Renal Disease. Cardiorenal Med. 2012 May;2(2):117-24.

27 Chen HY, Kao TW, Huang JW, Tsai TJ, Wu KD. Association between dyslipidemia and residual renal function in patients on chronic peritoneal dialysis. Clin Nephrol. 2008 Sep; 70(3):233-9.

28 Lin YC, Lin YC, Peng CC, Chen KC, Chen $\mathrm{HH}$, Fang TC, et al. Effects of Cholesterol Levels on Mortality in Patients with Long-Term Peritoneal Dialysis Based on Residual Renal Function. Nutrients. 2018 Mar;10(3):300.

29 Sezer S, Tutal E, Arat Z, Akçay A, Celik H, Ozdemir FN, et al. Peritoneal transport status influence on atherosclerosis/inflammation in CAPD patients. J Ren Nutr. 2005 Oct;15(4): 427-34.

30 Ilhan F, Kalkanli ST. Atherosclerosis and the role of immune cells. World J Clin Cases. 2015 Apr;3(4):345-52.
31 Lee SH, Kang HY, Kim KS, Nam BY, Paeng J, Kim S, et al. The monocyte chemoattractant protein-1 (MCP-1)/CCR2 system is involved in peritoneal dialysis-related epithelial-mesenchymal transition of peritoneal mesothelial cells. Lab Invest. 2012 Dec;92(12):1698-711.

32 Kosmas CE, Silverio D, Tsomidou C, Salcedo MD, Montan PD, Guzman E. The Impact of Insulin Resistance and Chronic Kidney Disease on Inflammation and Cardiovascular Disease. Clin Med Insights Endocrinol Diabetes. 2018 Aug; 11:1179551418792257.

33 Caligiuri G, Rudling M, Ollivier V, Jacob MP, Michel JB, Hansson GK, et al. Interleukin-10 deficiency increases atherosclerosis, thrombosis, and low-density lipoproteins in apolipoprotein E knockout mice. Mol Med. 2003 Jan-Feb;9(1-2):10-7.

34 Pinderski Oslund LJ, Hedrick CC, Olvera T, Hagenbaugh A, Territo M, Berliner JA, et al. Interleukin-10 blocks atherosclerotic events in vitro and in vivo. Arterioscler Thromb Vasc Biol. 1999 Dec;19(12):2847-53.

35 Cawthorn WP, Sethi JK. TNF-alpha and adipocyte biology. FEBS Lett. 2008 Jan;582(1): $117-31$.

36 Chen X, Xun K, Chen L, Wang Y. TNF-alpha, a potent lipid metabolism regulator. Cell Biochem Funct. 2009 Oct;27(7):407-16.

37 Espinoza M, Aguilera A, Auxiliadora Bajo M, Codoceo R, Caravaca E, Cirugeda A, et al. Tumor necrosis factor alpha as a uremic toxin: correlation with neuropathy, left ventricular hypertrophy, anemia, and hypertriglyceridemia in peritoneal dialysis patients. Adv Perit Dial. 1999;15:82-6.

38 Zareie M, De Vriese AS, Hekking LH, ter Wee PM, Schalkwijk CG, Driesprong BA, et al. Immunopathological changes in a uraemic rat model for peritoneal dialysis. Nephrol Dial Transplant. 2005 Jul;20(7):1350-61.

39 Burdeyna O, Stepanova N, Driyanska V. MCP-1 intraperitoneal production is associated with the peritoneal dialysis adequacy and technique survival. Nephrol Dial Transplant. 2017;32(3 suppl_3):iii637.

40 Stepanova N, Burdeyna O, Driyanska V, Savchenko V, Kolesnyk M. Systemic effects of local production of pro- and anti-fibrogenic mediators in peritoneal dialysis patients. Ukr J Nephrol Dial. 2016;4(52):52-8.

41 Zhou L, Wen F, Chen G, Liu J, Liu H, Peng Y, et al. Cytokine profiles in peritoneal dialysis effluent predicts the peritoneal solute transport rate in continuous ambulatory peritoneal dialysis patients. Int J Clin Exp Med. 2015 Nov; 8(11):20424-33.

42 Sach M, Bauermeister K, Burger JA, Loetscher $\mathrm{P}$, Elsner J, Schollmeyer P, et al. Inverse MCP$1 / \mathrm{IL}-8$ ratio in effluents of CAPD patients with peritonitis and in isolated cultured human peritoneal macrophages. Nephrol Dial Transplant. 1997 Feb;12(2):315-20. 